\title{
Optical Carrier Source for the Wavelength-Division-Multiplex-Radio-over-Fiber System Based on the Superstructure Fiber Bragg Grating
}

\author{
Hongjun Yang \\ School of Electronic Information Engineering \\ Chengdu University \\ Chengdu, China \\ e-mail: yanghongjun@cdu.edu.cn
}

Yongliang Liu

Chengdu Research Institute

Huawei Technologies Co.,Ltd

Chengdu, China

e-mail: ly.liang81@gmail.com

\author{
Yu Wang \\ Guangdong Planning and Designing Institute of \\ Telecommunications Co..Ltd \\ Guangzhou, China \\ e-mail: wangyu@gpdi.com \\ Rui Liu \\ Guangdong Planning and Designing Institute of \\ Telecommunications Co..Ltd \\ Guangzhou, China \\ e-mail: liur@gpdi.com
}

\begin{abstract}
Spectral slicing of a supercontinuum source has a good prospect in the applications of the wavelength-division-multiplexing (WDM)radio-over-fiber(RoF) systems. A comb filter based on superstructure fiber Bragg grating (SSFBG) to slice the supercontinuum spectrum generated in the photonic crystal fiber is designed and experimentally demonstrated. This method provides a low cost solution for WDM-RoF optical source.
\end{abstract}

Keywords-supercontinuum; wavelength division multiplex (WDM); radio over fiber (RoF); superstructure fiber Bragg grating (SSFBG)

\section{INTRODUCTION}

The initial purpose of Radio over Fiber (RoF) technology is to improve the utilization of spectrum resources and reduce the cost of remote base station modules. With the continuous development of research on RoF, the advantages of communication system based on RoF technology (such as rich in transmission bandwidth, seamless coverage, large capacity, low loss, etc.) is more and more obvious, which has great development space in the $3 \mathrm{G}$ and $4 \mathrm{G}$.

In optical communication, wavelength division multiplexing (WDM) technology has been very mature. It not only exists no technical problems, but also can simplify the network structure that multiplexing the multi-channel RoF signals and transmitting in one fiber. We call it WDM-RoF system[1,2].

The spectral slicing method of supercontinuum spectrum generated in the photonic crystal fiber is studied in this paper. The comb filter based on the superstructure fiber Bragg grating (SSFBG) is designed for the supercontinuum spectrum slicing. And the results are verified by experiment.

\section{GNERATION OF SUPERCONTINUUM SPECTRUM}

If in RoF system the two mixing-frequency optical waves are from different lasers, with the laser frequency jitter, the frequency difference of the two optical waves will change and cause the phase noise, which has great impact on the performance of millimeter wave signals. However, the advantage of the wavelength interval stability of the supercontinuum spectrum slicing will greatly reduce the phase noise in RoF system. Especially as the cost of the supercontinuum spectrum slicing technology is relatively low, it is a very promising scheme for a multi-channel signal source[3].

The generated supercontinuum spectrum is shown in Fig. 1. The spectrum bandwidth is about $80 \mathrm{~nm}$, and the flat area is from $1545 \mathrm{~nm}$ to $1575 \mathrm{~nm}$. For the 10 channels WDM-RoF system in the experiment, the bandwidth is about $10 \mathrm{~nm}$. So the generated supercontinuum spectrum meets the research needs.

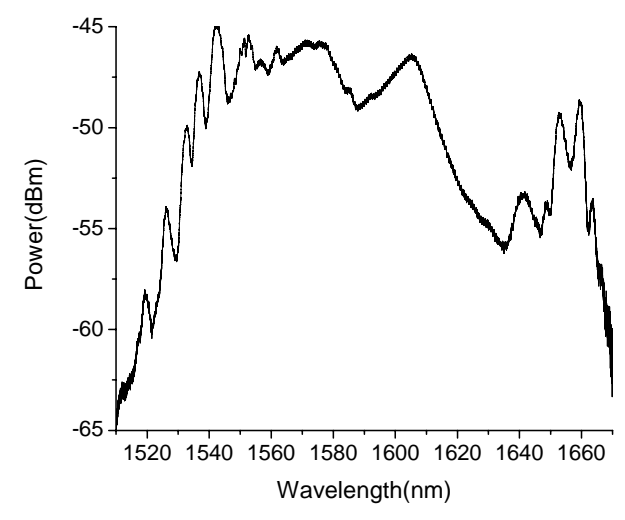

Figure 1. Test graph of SC spectrum 


\section{DESIGN AND EXPERIMENT OF SSFBG FILTER}

\section{A. SSFGB Filter}

The refractivity change of uniform fiber Bragg grating (UFBG) can be regarded as a perturbation added to the refractivity of the ordinary fiber core, $n_{\text {eff }}$, the distribution of which is[1,4]

$$
\delta n_{\text {eff }}(z)=\overline{\delta n}_{\text {eff }}(z)\left(1+v \cos \frac{2 \pi}{\Lambda} z\right)
$$

In (1), $\overline{\delta n}_{\text {eff }}(z)$ is the average change of fiber core refractivity, $\Lambda$ is the period of grating, $v$ is the fringe visibility, normally equal to 1 .

The periodical arrangement of fiber grating in time domain or space domain will make the spectrum discrete. SSFBG is to achieve the comb filtering effect by inserting a blank fiber (sampling fiber grating) or a phase-shifted fiber grating between each UFBG. In the experiment we take a sampling fiber grating as the grating filter.

The influence on SSFBG spectrum the other parameters of sampling fiber grating have is mainly manifested in the changes of amplitude and interval of reflection peak. The parameters affecting the reflection peak amplitude are mainly the sub-grating length, the refractivity modulation of fiber core $\overline{\delta n}_{\text {eff }}$ and the spatial sampling period of grating $N$. The increase of the three parameters can make the amplitude of reflection peak increase.

Studying the parameters affecting the reflection peak interval of sampling fiber grating, from (1), according to the Bragg formula of fiber grating, we can get[5]

$$
\Delta \lambda=\frac{\lambda_{B}^{2}}{2 n_{e f f} p}
$$

In (2), $p$ is the length of sampling period, $\Delta \lambda$ is the reflection peak interval. The peak interval, $\Delta \lambda$, is inversely proportional to $n_{\text {eff }}$ and $p$, and proportional to $\lambda_{B}$.

Because filtering the supercontinuum spectrum from the same optical source, the wavelengths of each filtered optical wave are strictly correlative and the phase noise is the same, then the difference frequency signal will not produce the phase noise with the temperature drift of optical source. Therefore according to the requirements of WDM-RoF, as long as the reflection peak intervals of sampling fiber grating are fixed from $40 \mathrm{GHz}$ to $100 \mathrm{GHz}$, the millimeter wave signals with good performance can be got in RoF base station.

\section{B. Design and Experiment}

SSFBG can be regarded as composed of $N$ $(l=0,1,2, \ldots, N-1)$ periods in a cascading style. Each period consists of a length of uniform sub- grating, $a$, and a length of blank fiber, $b$. The length of each period, $p=a+b$. The total length of SSFBG, $L=N p$, as shown in Fig. 2. Analysis of SSFBG characteristics using the transfer matrix method, suppose the forward transmission mode and the reverse transmission mode of optical field are respectively,
$R(z)$ and $S(z)$, the transfer matrix of each sub-grating multiplied by the transfer matrix of each blank fiber, we can obtain the transfer matrix of each period, then get the transfer matrix of the whole SSFBG.

The effective refractivity of fiber core, $n_{\text {eff }}$, are generally fixed in actual application, and 1310nm and $1550 \mathrm{~nm}$ are often selected as the transmission bands. So according to (2), the key parameter of determining the reflection peak interval is the period of grating, $p$.

Taking $p=2.59 \mathrm{~mm}, \quad n_{\text {eff }}=1.446, \quad v=1, \overline{\delta n}_{\text {eff }}=10^{-4}$, $\lambda_{B}=1550 \mathrm{~nm}$, the quantities of sub-grating $N=20$, the duty ratio $(a / p) S=0.05$, we can get the SSFBG simulation when the reflection peak interval is $40 \mathrm{GHz}$.

In Fig. 3 we can see there are about 20 reflection peaks the reflectivity of which is greater than $90 \%$. It meets the need of the design. Fig. 4 shows the local enlarged detail. The peak wavelengths are respectively $1549.006 \mathrm{~nm}$ and $1549.326 \mathrm{~nm}$, and the peak interval is $0.32 \mathrm{~nm}$.

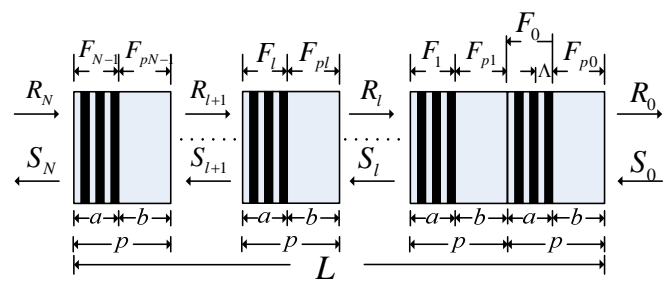

Figure 2. Transfer matrix module of SSFBG

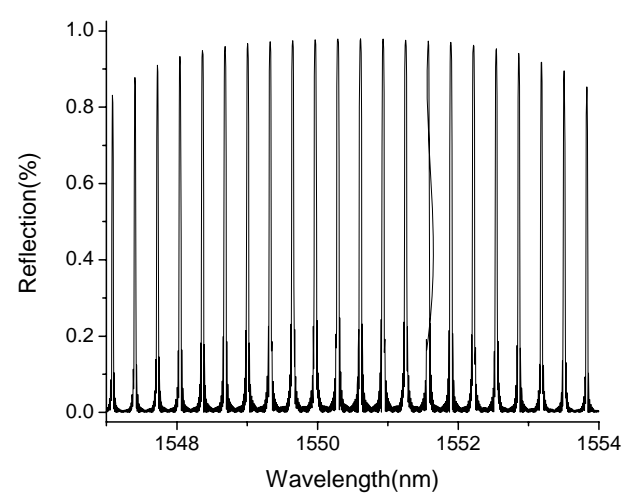

Figure 3. SSFBG reflection spectrum simulation 


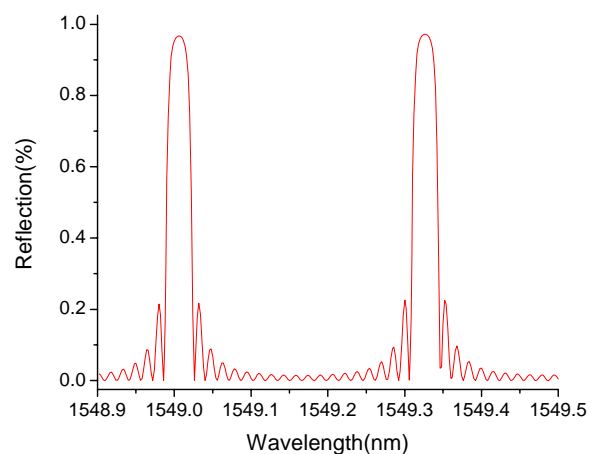

Figure 4. Local enlarged detail of SSFBG simulation

\section{Making the SSFBG Filter}

Fig. 5 and Fig. 6 respectively show the experimental graph and the theoretical graph of SSFBG when the peak interval is $0.8 \mathrm{~nm}(100 \mathrm{GHz})$. According to the two figures, we can see that compared the experimental result with the theoretical result, the peak of the simulation result is relatively narrow. But their peak intervals maintain a good agreement.

Fig. 7 shows the experimental result of supercontinuum spectrum filtered through SSFBG. Compared with Fig. 6, the distance between the reflection peaks is unchanged in Fig. 7. Because of the relative unflatness of supercontinuum spectrum, the peaks fluctuate. But it does not affect the performance of RF signals generated in the RoF base station.

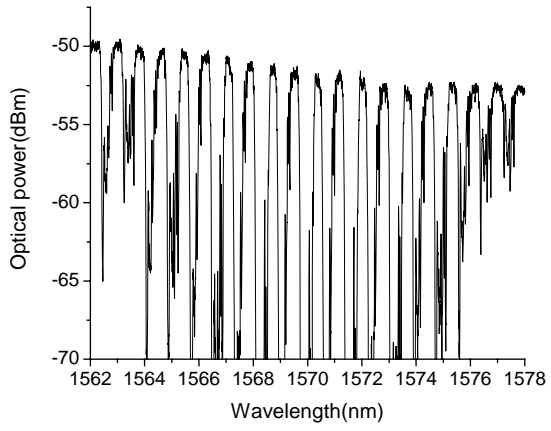

Figure 5. Experimental result of SSFBG

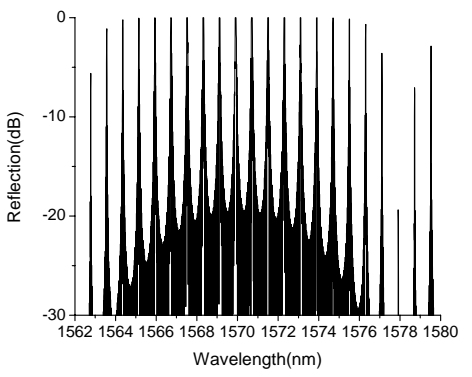

Figure 6. Simulation result of SSFBG

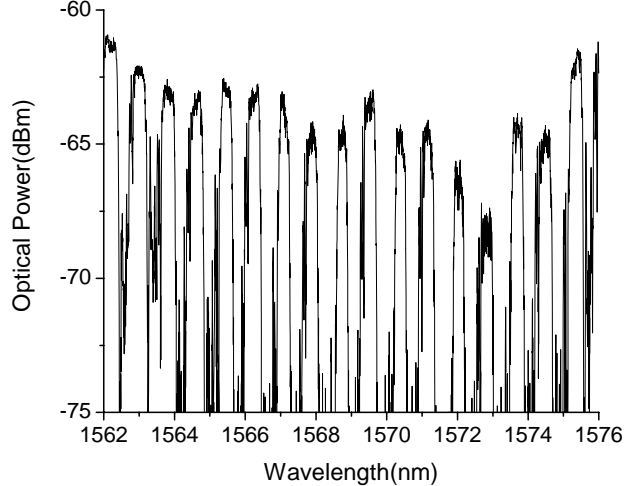

Figure 7. Experimental result of SC spectrum

\section{ANALYSIS AND DISCUSSION}

According to the experiment and simulation, the SSFBG can be used for optical heterodyne OCS modulation of RoF and the peak interval is $0.32 \mathrm{~nm}$. Among 20 reflection peaks the reflectivity difference doesn't exceed $10 \%$. The increase of $\overline{\delta n}_{\text {eff }}$ can cause the peak reflectivity grows, the flatness is better, but the line width increases. The increase of the quantities of grating can also cause the increase of the reflectivity but doesn't affect the line width. On the condition that the duty ratio doesn't change, the increase of the period length of sub-grating can cause the spectral envelope becomes sharper and the line width reduces. These results are verified by experiments.

Comparing the experiment with the simulation of SSFBG, the consistency of their peak interval means this design scheme is feasible. While the millimeter wave signals are generated in RoF base station, as there is no phase noise, the millimeter wavelength from mixing the frequencies is the wavelength difference of two optical waves, which is also the wavelength difference of the corresponding reflection peaks. The experimental results fulfill the actual requirements.

In order to reduce the influence of external conditions on SSFBG, SSFBG should not be too long. And when applicated in $40 \mathrm{GHz}$ or below $40 \mathrm{GHz}$ band, the peak intervals will be smaller. In frequency domain the increase of sampling frequency corresponds to the increase of sampling period in time (or spatial) domain. If we need to keep the same reflectivity, we have to increase the length of sampling fiber grating. If the phase shift is added periodically on sampling fiber grating, the reflection peak intervals can be reduced under the condition that the total length of the fiber is unchanged.

\section{CONCLUSIONS}

In this paper, the supercontinuum spectrum is generated in the normal dispersion region of photonic crystal fiber. The SSFBG comb filter is designed for the optical source of WDM-RoF and the results are verified by experiments. Finally the effect of some factors on the properties of 
SSFBG is discussed. Using SSFBG to generated supercontinuum spectrum in photonic crystal fiber and spectrum slicing have good application prospects in the realization of the optical source of WDM-RoF system.

\section{ACKNOWLEDGMENT}

Thank Chengdu University Science \& Technology Fund (2012XJZ16).

\section{REFERENCES}

[1] Turan Erdogan, "Fiber grating spectral," J.Lightw.Technol., vol.15, pp. 1277-1294, August 1997

[2] W.W.Hu, K.Inagaki, and T.Tanaka, "Millimetre-wave band $(50 \mathrm{GHz})$ multi-carrier generation using injection-locking technique for radio-over-fibre WDM communication system,” IEE Electron.Lett., vol. 40, pp. 1505-1506, Dec. 2004

[3] J.Yu, Z.Jia, and L.Xu, "DWDM optical millimeter-wave generation for radio-over-fiber using an optical phase modulator and an optical interleaver," IEEE Photon.Technol.Lett., vol. 18, pp. 1418- 1420, June 2006

[4] S.Fukushima, C.F.C.Silva, and Y.Muramoto,”Optoelectronic millimeter-wave synthesis using an optical frequency comb generator, optically injection locked lasers, and a unitraveling-carrier photodiode,” J.Lightw. Technol., vol. 21, pp. 3043-3051, Dec. 2003

[5] T.Nakasyotani, H.Toda, and T. Kuri, "Wavelength-division-multiplexed millimeter-waveband radio-on-fiber system using a supercontinuum light source," J. Lightw.Technol., vol.24, pp. 404-411, Jan. 2006 